\title{
The effect of two different aerobic training modalities in a child with spinal muscular atrophy type II: a case report
}

\author{
Numan Bulut',* , Bilge Nur Yardimci', Ender Ayvat', Orkun Tahir Aran², Öznur Yilmaz', Ayşe Karaduman \\ 'Department of Physiotherapy and Rehabilitation, Faculty of Health Sciences, Hacettepe University, Ankara, Turkey \\ ${ }^{2}$ Department of Occupational Therapy, Faculty of Health Sciences, Hacettepe University, Ankara, Turkey
}

This study examined the effect of two different aerobic training modalities in a boy with spinal muscular atrophy type II. Motor functions were measured with Hammersmith Functional Motor Scale (HFMS) and Gross Motor Function Measure (GMFM). Spirometry was utilized for assessing pulmonary functions and PedsQL 3.0 Neuromuscular module was utilized for quality of life of child. Ergometer training was applied 3 times per week for the duration of 12 weeks. After 6 weeks of wash-out period, aquatherapy was applied for twice a week for the duration of 12 weeks. HFMS and GMFM scores, and pulmonary functions of the child and quality of life scores of his parents were improved during both mo- dalities. These improvements were largely maintained during 1-year follow-up. This study showed that both modalities had similar effects on our case's pulmonary, motor functions and quality life of himself and parents. It will be a guide for researchers working in the area of children with neuromuscular disorders regarding plan and diversification of therapy program.

Keywords: Aquatherapy, Ergometer training, Motor function, Quality of life, Respiratory function, Spinal muscular atrophy

\section{INTRODUCTION}

Spinal muscular atrophy (SMA) is a neuromuscular disorder, characterized by autosomal recessive inherited mutation in survival motor neuron gene (SMN1). Mutation in SMN1 causes degeneration of anterior horn motor neuron and leads to hypotonia, muscular weakness and atrophy (D'Amico et al., 2011). In SMA type II, symptoms such as disability of head control and hypotonia show up before 18 months of age. Although children are able sit and stand independently, they are unable to walk. Also, pulmonary complications and dysphagia commonly are seen (Kroksmark et al., 2001; Wang et al., 2007).

Until recently, there was no clear treatment of the disease, while nusinersen, an antisense oligonucleotide, was approved by both U.S. Food and Drug Administration and EMA for all types of SMA in the last year. In a published study regarding the results of the clinical trial, this oligonucleotide was found out to be effective in $51 \%$ of patients with SMA type I (Finkel et al., 2017). Cur- rently nusinersen is only administered for SMA type I in Turkey. However physiotherapy, diet, pulmonary support and drug treatments are applied for the symptom control (Wang et al., 2007). The goal of physiotherapy is to protect the functionality of children at the highest level by minimizing the symptoms of the disease. Although there are studies in which different sub-parameters of aerobic training are used, such as hydrotherapy and ergometer training in adult SMA type III who were able to walk, there are limited studies about the children with type II due to the progressive nature and rareness of the disease (Madsen et al., 2015, Salem and Gropack, 2010). In these studies, generally the effects on motor function were investigated. Our study hypothesis was that aquatherapy was more effective than ergometer training on improving motor and pulmonary function and quality of life.

The aim of this study was to determine the effect of two different aerobic training modalities on pulmonary functions and quality of life as well as motor function in a child with SMA type II.
${ }^{\star}$ Corresponding author: Numan Bulut (D) https://orcid.org/0000-0001-5427-1103 Department of Physiotherapy and Rehabilitation, Faculty of Health Sciences, Hacettepe University, 06100, Altındağ, Ankara, Turkey

E-mail: nmn60_90@hotmail.com

Received: December 30, 2018 / Accepted: January 8, 2019
This is an Open Access article distributed under the terms of the Creative Commons Attribution Non-Commercial License (http://creativecommons.org/licenses/by-nc/4.0/) which permits unrestricted non-commercial use, distribution, and reproduction in any medium, provided the original work is properly cited. 


\section{MATERIALS AND METHODS}

\section{Case description}

A boy with the age of 60 months was included to the study. His body mass index (BMI) was $18.14 \mathrm{~kg} / \mathrm{m}^{2}$ (weight, $20 \mathrm{~kg}$; height, $105 \mathrm{~cm}$ ). The child was carried to 39 weeks of pregnancy, born via cesarean delivery, and weighed $3.4 \mathrm{~kg}$ at birth. There is no family history of SMA. Although the child was able to sit at 6 months, family applied to hospital at 16 months of age due to child's lack of ability to stand and walk, hypotonia and general weakness of the limbs and body. After the child was examined by a pediatric neurologist and a referral was made for a genetics consultation. The DNA analysis revealed that homozygous deletion of exon 7-8 region in the SMN1 gene and two copies of the SMN2 at 18 months. He has received conventional physiotherapy approaches (stretching, range of motion, trunk and upper extremity strengthen and standing exercise with support etc.) upon diagnosis. Because of frequent pulmonary infections at the age of $3,2.5$ $\mathrm{mg}$ of ventolin was prescribed daily for the duration of 6 months. The main symptoms of the child were that he lacked head control and was unable to stand before he began his physical therapy sessions. The expectation of investigators was to minimize these symptoms after ergometer training and aquatherapy. The child and his parent expectations were the same. Written informed consents were granted from the boy and his parents.

\section{Examination}

Goniometric measurements of shortness and joint limitation, as well as the following physiotherapy assessments were applied on initial assessment and end of training. Also follow-up assessments were carried out 1 year after aquatherapy.

\section{Motor function}

Motor functions were measured with the Hammersmith Functional Motor Scale (HFMS) and Gross Motor Function Measure (GMFM-88). HFMS was developed by Main et al. (2003) to examine motor skills and clinical course of SMA type II and III children. The scale consists of 20 items evaluating functions such as rolling, sitting and standing. Each item is scored between 0 and 2 points, and maximum score is 40 . There is a correlation between scores and motor skills, higher score means better motor skills.

GMFM-88 was developed to evaluate gross functions in children with cerebral palsy (Russell et al., 1989). The GMFM-88 scale is also a valid and sensitive outcome measure for children with SMA (Nelson et al., 2006). The GMFM-88 items are divided into 5 subparameters (lying and rolling; sitting; crawling and kneeling; standing; and walking, running and jumping). Each item is scored as 0 (does not initiate), 1 (initiates), 2 (partially completes), and 3 (completes). Total score is given as a percentage and a healthy 5 -years-old is expected to take the full score (100\%) on this scale.

\section{Pulmonary function}

The pulmonary functions of the child were measured using a Spirobank G USB device and the data was transferred to the computer via Winspro software. Pulmonary function test was performed in compliance with American Thoracic Society guidelines (Miller et al., 2005). The child was asked to perform the test for three times with maximum effort. The highest forced vital capacity (FVC), forced expiratory volume in $1 \mathrm{sec}\left(\mathrm{FEV}_{1}\right), \mathrm{FVC} / \mathrm{FEV}_{1}$ and peak expiratory flow values were recorded. The child was motivated before each evaluation by saying that there were different animals on the back of the screen on the computer, the stronger and longer the expiration or the more he coughed and the more he could see the animals.

\section{Quality of life}

The PedsQL 3.0 neuromuscular module was completed both by child and parents to assess the quality of life of the child. The questionnaire can be used in 4 different age groups which are 2-4, 5-7, 8-12, and 13-18 years old. The child self-report consists of 17 items and the parent proxy-report has 25 items. There are expressions in the both report as 'It is hard to breathe'. Each expression scores 0 (never), 2 (sometimes), and 4 (always) in the child self-report. In the parent proxy-report, each item is scored between 0 (never) and 4 (always). High scores show higher quality of life (Iannaccone et al., 2009).

\section{Interventions}

\section{Ergometer training}

Ergometer training was held 3 times a week for 12 weeks in the clinic under physiotherapist supervision. Ergometer training was given with MOTOmed viva2 device (Betzenweiler, Germany). The MOTOmed viva2 device provides visual feedback about child's cycling motion whether it is passive or active, symmetrical. Cycling ergometer training is given at $60 \%-70 \%$ of maximum heart rate measured by a pulse oximeter. Each session consisted of a 5-min warm-up, 20-min active cycle ergometer and a 5-min cooling period. After the $5 \mathrm{~min}$ of resting period, the child contin- 
ued the same protocol for arm-cycling ergometer training at $60 \%-70 \%$ of the maximal heart rate. He sat on a height adjustable chair with back support during training.

\section{Aquatherapy}

A 6 weeks of wash-out period was given after ergometer training. Then the Halliwick method, was administered 45 min twice a week for the duration of 12 weeks. The aquatherapy was administered in a $7.5-\mathrm{m} \times 25-\mathrm{m}$ therapy pool. The height of therapy pool $140 \mathrm{~cm}$ and water temperature was $30^{\circ} \mathrm{C}-32^{\circ} \mathrm{C}$. Arm and leg cuff floatation devices were used for the child's safety. The parent was present during the therapy sessions to enhance the child's motivation and minimize his fear of water. Therapy consists of 5-10 min of warm-up (such as breathing and walking [with the help of elevation]) and stretching exercises, 40 min of Halliwick concept (Martin, 1981) (lying in different directions, rolling and swimming) and 5-10 min of cool-down exercises (walking).

He continued to receive the conventional physiotherapy (stretching, range of motion, trunk and upper extremity strengthen and standing exercise with support etc.) 3 times per week during ergometer training, wash-out period and aquatherapy session. Patient did not take any medication during exercise training periods.

\section{RESULTS}

BMI after ergometer training was 17.35 , while before aquatherapy it was 17.07 and $16.74 \mathrm{~kg} / \mathrm{m}^{2}$ after aquatherapy. BMI of follow-up was $18.90 \mathrm{~kg} / \mathrm{m}^{2}$. In the result of goniometric assessment, right hip flexor muscles and bilateral hamstring shortening were determined. In addition, $8^{\circ}$ and $9^{\circ}$ limitation found at the right and left ankle joint, consecutively. There was no change in short- ness and joint limitation values during treatment sessions and follow-up.

After ergometer training and aquatherapy, GMFM total scores were improved by 13.37 and 11.04, successively. HFMS score was increased by five points after ergometer training and three points after aquatherapy. The motor function changes are shown in Fig. 1. Changes in pulmonary functions and the quality of life values of the child and also his parent values are shown in Table 1. These gains were largely maintained during follow-up period.

After aquatherapy, his parents addressed questions such as what kind of training is more effective, and whether side effect was experienced during the training. His mother stated that two different aerobic training improved general function as well as especially leg function and there is no side effect. It was also stated that the child is more willing to participate in aquatherapy and has greater confidence that he will develop his movements after aquatherapy sessions.

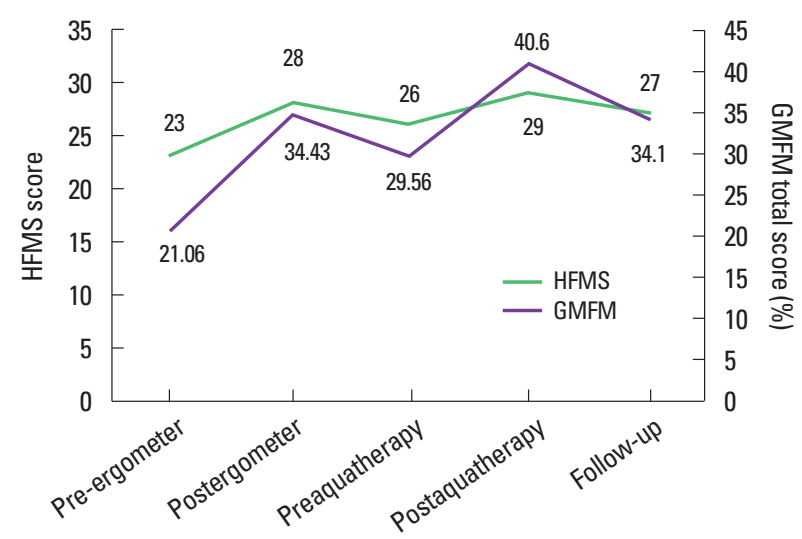

Fig. 1. Hammersmith Functional Motor Scale (HFMS) score and Gross Motor Function Measure (GMFM) total score of the child.

Table 1. Pulmonary functions and quality of life scores of the child and his parents

\begin{tabular}{|c|c|c|c|c|c|c|c|c|c|c|}
\hline \multirow{2}{*}{ Parameter } & \multicolumn{2}{|c|}{ Pre-ergometer training } & \multicolumn{2}{|c|}{ Postergometer training } & \multicolumn{2}{|c|}{ Preaquatherapy } & \multicolumn{2}{|c|}{ Postaquatherapy } & \multicolumn{2}{|c|}{ Follow-up } \\
\hline & Measured & $\%$ & Measured & $\%$ & Measured & $\%$ & Measured & $\%$ & Measured & $\%$ \\
\hline \multicolumn{11}{|l|}{ Pulmonary function } \\
\hline $\mathrm{FVC}(\mathrm{L})$ & 1.45 & 157 & 1.44 & 128 & 1.53 & 126 & 1.62 & 133 & 1.59 & 119 \\
\hline $\mathrm{FEV}_{1}(\mathrm{~L})$ & 1.31 & 155 & 1.36 & 134 & 1.47 & 136 & 1.51 & 139 & 1.44 & 121 \\
\hline $\mathrm{FEV}_{1} / \mathrm{FVC}(\%)$ & 90.30 & 96 & 94.40 & 101 & 96.00 & 103 & 93.20 & 100 & 90.50 & 97 \\
\hline $\mathrm{PEF}(\mathrm{L} / \mathrm{sec})$ & 2.40 & 303 & 2.54 & 215 & 2.56 & 169 & 2.70 & 178 & 2.75 & 143 \\
\hline \multicolumn{11}{|l|}{ Quality of life } \\
\hline Parents proxy-report & \multicolumn{2}{|c|}{40.00} & \multicolumn{2}{|c|}{55.00} & \multicolumn{2}{|c|}{39.00} & \multicolumn{2}{|c|}{59.00} & \multicolumn{2}{|c|}{55.00} \\
\hline Child self-report & \multicolumn{2}{|c|}{61.76} & \multicolumn{2}{|c|}{52.94} & \multicolumn{2}{|c|}{70.58} & \multicolumn{2}{|c|}{73.52} & \multicolumn{2}{|c|}{55.88} \\
\hline
\end{tabular}

FVC, forced vital capacity; FEV 1 , forced expiratory volume in $1 \mathrm{sec}$; PEF, peak expiratory flow. 


\section{DISCUSSION}

In this case report, the effects of 12 weeks of ergometer and 12 weeks of aquatherapy programs have been compared and it has shown that all parameters recorded, except for the quality of life of the child, introduced positive changes and these changes were shown to be largely maintained in the follow-up process.

Although there is no study regarding ergometer training with children with SMA, there is a study carried out regarding ergometer training in the adults with SMA (Salem and Gropack, 2010). In the study carried out by Madsen et al. (2015) it has stated that cycling ergometer training conducted on 6 SMA type III and 9 healthy children, increases the maximal oxygen consumption in SMA type III children without forming muscle damage, but it is not effective in the function and caused fatigue. In a study in which the effects of a bicycle ergometer training in eight adults with spinal and bulbar muscular atrophy were investigated (Preisler et al., 2009), it has determined that maximal workload and citrate synthesis have been increased but no improvement is observed in maximal oxygen consumption and daily living activities. Contrary to the studies conducted, after the ergometer training it has been shown that SMA type II children develop gross motor function and positive effects on life quality of child from parent's aspect in this case report. It is considered that the reason of development in the gross motor function, quality of life is that this study investigated the children patients on their early age comparing the other studies.

SMA motor development is characterized with progressive muscle weakness. In the study of the effectiveness of aquatherapy in patients with SMA types II and III who received treatment according to Halliwick method, significant improvements in motor activities of daily living were recorded (Cunha et al., 1996; Getz et al., 2006). In two separate case reports with children with SMA types I and III, hydrotherapy training has been shown to improve gross motor functions and gait parameters (Figuers, 1999; Salem and Gropack, 2010). In this case report, after both ergometer training and aquatherapy, it has been observed that there has been development in the HFMS and GMFM. In all types of SMA (I, II, and III), aquatherapy applied on early period have positive effects on motor performance. In addition to positive aspects of aquatherapy, it has been shown that both the pulmonary functions are preserved and the parents improve their quality of life in this case report. However, it is thought that the abnormal variability in the quality of life of the child is due to the age of the child. There are no studies evaluating the pulmonary function after aquatherapy in children with SMA. However, due to the hydrostatic pressure of the water, it is thought that aquatherapy affects the pulmonary functions positively. In our study, the fact that pulmonary functions after aquatherapy were preserved in spite of minimal developments, indicates that the training would be beneficial for children with SMA. What limits this study is that the data cannot be generalized as with all case reports and exercise capacity (etc. maximal workload and $\mathrm{O}_{2}$ uptake) was not assessed.

Although it is not possible to generalize the case results, it has shown that both ergometer training and aqutherapy are effective on motor functions and quality of life and can be used safely among children with SMA type II, as in other types of SMA. Although selection of aerobic training depends on the preferences and socio-economic status of their family, in our case, parents and the child preferred aquatherapy. These results indicate that there is an improvement of motor and pulmonary functions and quality of life as well as the preservation of the gain for 1 year will be a guide for larger and controlled studies for researchers working in this area. Also, these kinds of improvements in this disease characterized by progressive muscle weakness have once again emphasized the effectiveness of aerobic training. According the results of medical study carried out among the patients with SMA type I, it has shown that the effect of medication the children who received medication on the functionally high level, is higher comparing the others (Finkel et al., 2017). The possibility of gaining the higher medication effect in the future clinical studies among the other SMA patients in addition to SMA type I, and the high possibility of including the patients which are on the high functional level to these studies have once again emphasized the utmost importance of alternative methods such as aerobic in this field.

\section{CONFLICT OF INTEREST}

No potential conflict of interest relevant to this article was reported.

\section{ACKNOWLEDGMENTS}

Thanks to Sibel Aksu Yıldırım for her assistance with setting up the therapy pool and İpek Alemdaroğlu for her assistance with this study.

\section{REFERENCES}

Cunha MC, Oliveira AS, Labronici RH, Gabbai AA. Spinal muscular atro- 
phy type II (intermediary) and III (Kugelberg-Welander). Evolution of 50 patients with physiotherapy and hydrotherapy in a swimming pool. Arq Neuropsiquiatr 1996;54:402-406.

D’Amico A, Mercuri E, Tiziano FD, Bertini E. Spinal muscular atrophy. Orphanet J Rare Dis 2011;6:71.

Figuers C. Aquatic therapy intervention for a child diagnosed with spinal muscular atrophy. Phys Ther Case Rep 1999;2:109-112.

Finkel RS, Mercuri E, Darras BT, Connolly AM, Kuntz NL, Kirschner J, Chiriboga CA, Saito K, Servais L, Tizzano E, Topaloglu H, Tulinius M, Montes J, Glanzman AM, Bishop K, Zhong ZJ, Gheuens S, Bennett CF, Schneider E, Farwell W, De Vivo DC; ENDEAR Study Group. Nusinersen versus sham control in infantile-onset spinal muscular atrophy. N Engl J Med 2017;377:1723-1732.

Getz M, Hutzler Y, Vermeer A. Effects of aquatic interventions in children with neuromotor impairments: a systematic review of the literature. Clin Rehabil 2006;20:927-936.

Iannaccone ST, Hynan LS, Morton A, Buchanan R, Limbers CA, Varni JW; AmSMART Group. The PedsQL in pediatric patients with Spinal Muscular Atrophy: feasibility, reliability, and validity of the Pediatric Quality of Life Inventory Generic Core Scales and Neuromuscular Module. Neuromuscul Disord 2009;19:805-812.

Kroksmark AK, Beckung E, Tulinius M. Muscle strength and motor function in children and adolescents with spinal muscular atrophy II and III. Eur J Paediatr Neurol 2001;5:191-198.

Madsen KL, Hansen RS, Preisler N, Thøgersen F, Berthelsen MP, Vissing J. Training improves oxidative capacity, but not function, in spinal muscular atrophy type III. Muscle Nerve 2015;52:240-244.
Main M, Kairon H, Mercuri E, Muntoni F. The Hammersmith functional motor scale for children with spinal muscular atrophy: a scale to test ability and monitor progress in children with limited ambulation. Eur J Paediatr Neurol 2003;7:155-159.

Martin J. The Halliwick method. Physiotherapy 1981;67:288-291.

Miller MR, Hankinson J, Brusasco V, Burgos F, Casaburi R, Coates A, Crapo R, Enright P, van der Grinten CP, Gustafsson P, Jensen R, Johnson DC, MacIntyre N, McKay R, Navajas D, Pedersen OF, Pellegrino R, Viegi G, Wanger J; ATS/ERS Task Force. Standardisation of spirometry. Eur Respir J 2005;26:319-338.

Nelson L, Owens H, Hynan LS, Iannaccone ST; AmSMART Group. The gross motor function measure is a valid and sensitive outcome measure for spinal muscular atrophy. Neuromuscul Disord 2006;16:374380.

Preisler N, Andersen G, Thøgersen F, Crone C, Jeppesen TD, Wibrand F, Vissing J. Effect of aerobic training in patients with spinal and bulbar muscular atrophy (Kennedy disease). Neurology 2009;72:317-323.

Russell DJ, Rosenbaum PL, Cadman DT, Gowland C, Hardy S, Jarvis S. The gross motor function measure: a means to evaluate the effects of physical therapy. Dev Med Child Neurol 1989;31:341-352.

Salem Y, Gropack SJ. Aquatic therapy for a child with type III spinal muscular atrophy: a case report. Phys Occup Ther Pediatr 2010;30:313-324.

Wang CH, Finkel RS, Bertini ES, Schroth M, Simonds A, Wong B, Aloysius A, Morrison L, Main M, Crawford TO, Trela A; Participants of the International Conference on SMA Standard of Care. Consensus statement for standard of care in spinal muscular atrophy. J Child Neurol 2007;22:1027-1049. 